This item was submitted to Loughborough's Research Repository by the author.

Items in Figshare are protected by copyright, with all rights reserved, unless otherwise indicated.

\title{
Improved thermoelectric generator performance using high temperature thermoelectric materials
}

PLEASE CITE THE PUBLISHED VERSION

http://dx.doi.org/10.4271/2017-01-0121

\section{PUBLISHER}

(C) SAE International

\section{VERSION}

AM (Accepted Manuscript)

\section{PUBLISHER STATEMENT}

This work is made available according to the conditions of the Creative Commons Attribution-NonCommercialNoDerivatives 4.0 International (CC BY-NC-ND 4.0) licence. Full details of this licence are available at: https://creativecommons.org/licenses/by-nc-nd/4.0/

\section{LICENCE}

CC BY-NC-ND 4.0

\section{REPOSITORY RECORD}

Yang, Zhijia, Jesus PradoGonjal, Matthew Phillips, Song Lan, Anthony Powell, Paz Vaqueiro, Min Gao, Richard Stobart, and Rui Chen. 2017. "Improved Thermoelectric Generator Performance Using High Temperature Thermoelectric Materials". figshare. https://hdl.handle.net/2134/24128. 


\title{
Improved Thermoelectric Generator Performance using High Temperature Thermoelectric Materials
}

\author{
Author, co-author (Do NOT enter this information. It will be pulled from participant tab in \\ MyTechZone) \\ Affiliation (Do NOT enter this information. It will be pulled from participant tab in MyTechZone)
}

\begin{abstract}
Thermoelectric generator (TEG) has received more and more attention in its application in the harvesting of waste thermal energy in automotive engines. Even though the commercial Bismuth Telluride thermoelectric material only have $5 \%$ efficiency and $250^{\circ} \mathrm{C}$ hot side temperature limit, it is possible to generate peak $1 \mathrm{~kW}$ electrical energy from a heavy-duty engine. If being equipped with $500 \mathrm{~W}$ TEG, a passenger car has potential to save more than $2 \%$ fuel consumption and hence $\mathrm{CO}_{2}$ emission reduction. TEG has advantages of compact and motionless parts over other thermal harvest technologies such as Organic Rankine Cycle (ORC) and TurboCompound (TC). Intense research works are being carried on improving the thermal efficiency of the thermoelectric materials and increasing the hot side temperature limit. Future thermoelectric modules are expected to have $10 \%$ to $20 \%$ efficiency and over $500^{\circ} \mathrm{C}$ hot side temperature limit. This paper presents the experimental synthesis procedure of both p-type and n-type skutterudite thermoelectric materials and the fabrication procedure of the thermoelectric modules using this material. These skutterudite materials were manufactured in the chemical lab in the University of Reading and then was fabricated into modules in the lab in Cardiff University. These thermoelectric materials can work up to as high as $500^{\circ} \mathrm{C}$ temperature and the corresponding modules can work at maximum $400^{\circ} \mathrm{C}$ hot side temperature. The performance loss from materials to modules has been investigated and discussed in this paper. By using a validated TEG model, the performance improvement using these modules has been estimated compared to commercial Bisemous Telluride modules.
\end{abstract}

\section{Introduction}

More and more research efforts have been invested in waste thermal energy recovery in automotive engine in endeavor to improve the fuel economy and reduce $\mathrm{CO}_{2}$ emissions. There are multiple choices of technologies for waste thermal energy recovery in automotive applications. Among them there are Mechanical Turbo-Compounding (MTC), electrical turbo-compounding (ETC), Thermoelectric Generator (TEG) and Organic Rankine Cycle (ORC) [1-5]. Each technique has its own advantages and disadvantages. It is also possible to use different harvest techniques within a same engine system. Among them only TEG can claim all the following three advantages: 1) without moving parts; 2 ) harvest the thermal energy from multiple locations; 3 ) potential to be integrated to aftertreatment system.

Page 1 of 7
The state-of art commercial Thermoelectric Module (TEM) are made from Bismuth Telluride thermoelectric material with maximum 5\% efficiency and $250^{\circ} \mathrm{C}$ hot side temperature limit. It was reported that the TEG made of these modules could generate peak $1 \mathrm{~kW}$ electrical energy from a heavy-duty engine [6]. There is conservative prediction that if a passenger car is equipped with a 500W TEG, a there is potential to save more than $2 \%$ fuel consumption and hence $\mathrm{CO}_{2}$ emission reduction [7]. Since the commercial TEMs have hot side temperature limit which is $250^{\circ} \mathrm{C}$, a by-pass solution was used in most experimental TEG project for automotive engine especially gasoline engine which has higher exhaust temperature than that of diesel engine [8-11]. However, this solution is not ideal as there will be a lot of thermal energy at high temperature escaped without any recovery. So that developing high temperature thermoelectric materials is important for automotive applications. Meanwhile, segmenting the elements within the TEM using different thermoelectric materials can further improve the TEG performance [12]. Future thermoelectric modules are expected to have $10 \%$ to $20 \%$ efficiency and over $500^{\circ} \mathrm{C}$ hot side temperature limit. The possible solution is using segmented thermoelectric elements which consist of low temperature (Bismush Telluride), mid-range (skutterudite) and high-range(half-Heuslers [26]) thermoelectric materials.

The efficiency of a TEM is determined by the materials that constitute the TEM. Materials performance is expressed in terms of the dimensionless figure-of-merit ZT, which is the ratio of electrical power to heat flux. High performance requires the unusual combination of a high electrical conductivity $(\sigma)$, usually associated with metallic phases, together with a high Seebeck coefficient (S) and low thermal conductivity ( $\kappa)$, typical in non-metallic systems [13,14]. Materials with the skutterudite crystal structure possess attractive transport properties and have a good potential to achieve high ZT values at high temperature $[15,16]$.

A thermoelectric module can either be run with an applied bias to use electrical energy to move heat across the module and create a temperature difference (Peltier mode), or to generate electrical power from a heat source by creating a temperature gradient across the module and harvest power from the resulting heat flow (Seebeck mode). It is this second mode which makes thermoelectric modules an attractive technology for energy recovery from heat sources such as automotive exhaust systems. Bass et al demonstrated a $1 \mathrm{~kW}$ bismuth telluride system [6] in the mid-1990s and subsequently many others have followed [17]. Although ring structured thermoelectric modules to generate power from radial sources have been 
demonstrated in the lab [18] and commercially [19], most modules are fabricated with a planar design with heat flow from one side of the plate to another.

The rest of the paper was organized as: The basic theory of TEG was given in Section 2 which was followed by the introduction of manufacture of skutterudite material in Section 3. The introduction of fabrication of TEM made from skutterudite materials was presented in Section 4 . The property loss caused by the module fabrication procedure was discussed in Section 5. Section 6 is about how the improved TEG performance using high temperature skutterudite materials was estimated using a validated TEG model. The summary and conclusions can be found in Section 7.

\section{Theory of Thermoelectric Generator}

A helpful analogy for understanding the workings of the thermoelectric module is to consider it as a 'thermal battery' [23]. The Seebeck voltage (related to the Seebeck coefficient $\alpha$ by the equation $\mathrm{V}=\alpha \Delta \mathrm{T}$,) may then be considered like an EMF, while the internal resistance of the module $\mathrm{R}$, similar to the internal resistance of a battery. When a load RL is placed across the thermoelectric module, the voltage VL generated across the load is given by;

$$
V_{L}=\alpha \Delta T \frac{R_{L}}{R+R_{L}}
$$

and the current IL by the equation;

$$
I_{L}=\frac{\alpha \Delta T}{R+R_{L}}
$$

For a given temperature difference, the peak power will be achieved when the external load matches the internal resistance of the module i.e., when $\mathrm{RL}=\mathrm{R}$ and the maximum power is given by;

$$
P_{\max }=\frac{(\alpha \Delta T)^{2}}{4 R}
$$

Module efficiency is an important parameter to consider, especially if there is limited heat available. The maximum conversion efficiency $\eta$ of the module is given by the equation

$$
\eta=\frac{P_{\max }}{\dot{Q}_{\text {hot }}}
$$

where $\dot{Q}$ hot is the heat flowing through the hot side of the module and the peak power is measured under load conditions with the system in thermal equilibrium. When the module is under open circuit conditions, the heat flow is given by the equation

$$
\dot{Q}_{h o t}=\kappa A \frac{\Delta T}{\mathrm{~L}},
$$

where $\mathrm{A}$ and $\mathrm{L}$ are the area and length of the thermoelectric legs respectively. Under load conditions $\dot{Q}$ hot becomes

$$
\dot{Q}_{h o t}=\alpha I T_{h o t}-\frac{1}{2} I^{2} R+\kappa A \frac{\Delta T}{L} .
$$

Substituting equation (6) into equation (5) and using the relation $\mathrm{ZT}=\alpha 2 \sigma \mathrm{T} / \kappa$, the efficiency can be written in terms of the dimensionless figure of merit

$$
\eta=\left(\frac{\Delta T}{T_{h}}\right) \frac{\sqrt{Z \bar{T}+1}-1}{\sqrt{Z \bar{T}+1}+T_{C} / T_{h}} .
$$

The efficiency is strongly dependent on a large $\Delta \mathrm{T}$ and also large average ZT. Skutterudites are designed to work in temperatures up to $600{ }^{\circ} \mathrm{C}$ which has the potential to be a highly efficient module.

\section{Manufacture of Skutterudite Material}

The manufacture of Skutterudite material has been carried out in the lab of chemistry department in the University of Reading, UK. The skutterudite materials was produced during the synthetic process and then was characterized to find out its real three thermoelectric properties during the characterization stage.

Figure 1 shows the crystal structure of a filled skutterudite. The skutterudite structure consists of a framework of stoichiometry M8X24, (M = transition metal, $\mathrm{X}=$ pnictogen atom) formed by corner-sharing MX6 octahedra, and contains larges cages that can be filled with a heavy filler atom, i.e. rare earth [20-22].

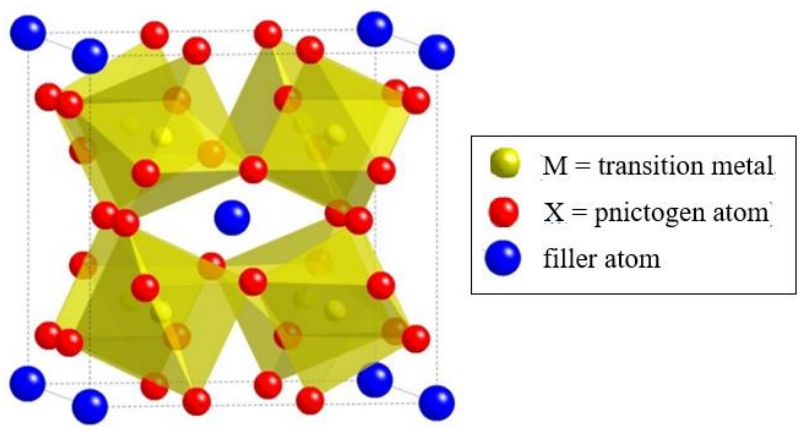

Figure 1. Crystal structure of a filled skutterudite. Transition metals are represented by yellow spheres, pnictogen atoms by red spheres and the filler atoms by blue spheres.

\section{Synthetic Procedures}

Pure elements of constituent elements were weighted according to the stoichiometric formula of $\mathrm{CoSb}_{2.75} \mathrm{Sn}_{0.05} \mathrm{Te}_{0.20}$ (n-type material) and $\mathrm{Ce}_{0.5} \mathrm{Yb}_{0.5} \mathrm{Fe}_{3.25} \mathrm{Co}_{0.75} \mathrm{Sb}_{12}$ (p-type material): The appropriate amounts of the powdered elements were loaded together with 13 Zirconium dioxide balls into a $250 \mathrm{ml}$ Zirconium dioxide grinding vessel. To prevent oxidation, the vessel was properly sealed inside an Argonfilled glove-box. The grinding vessel was loaded into a Fritsch P6 Planetary Ball Mill. Grinding was carried out at $400 \mathrm{rpm}$ for $10 \mathrm{~h}$. This methodology allows the preparation of $>60 \mathrm{~g}$ per batch at the laboratory scale. Figure 2 shows the Ball Mill device together with the grinding vessel. 
Ball Mill

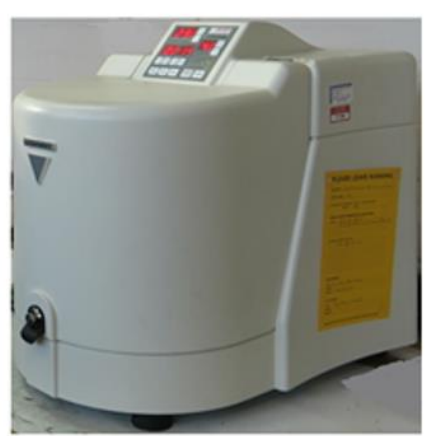

\section{Grinding Vessel}

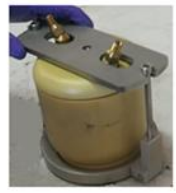

Inside the Grinding Vessel

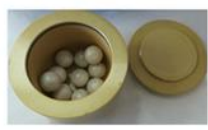

Figure 2. Ball Mill device and Grinding Vessel

The ball milled powders were consolidated by hot-pressing $(90 \mathrm{MPa}$, $600^{\circ} \mathrm{C}$ for 30 minutes). The densities of the consolidated discs were measured using an Archimedes balance ADAM PW184. The compacted samples have densities above $98 \%$ of the crystallographic values. The consolidated pellets show a dense microstructure, voidfree and with a relatively uniform particle size $(150-200 \mathrm{~nm})$. The pictures of skutterudite material powder and pellet made from this powder are shown in Figure 3.

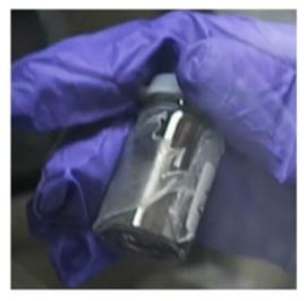

(a)
Mold for Hot Pressing

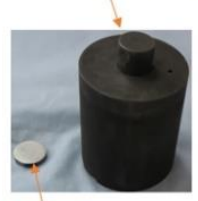

Pellet

(b)
Figure 3. (a) Manufactured skutterudite material power; (b) Skutterudite thermoelectric pellet.

\section{Characterization techniques}

\section{X-Ray diffraction}

X-ray diffraction (XRD) data for both $\mathrm{CoSb}_{2.75} \mathrm{Sn}_{0.05} \mathrm{Te}_{0.20}$ and $\mathrm{Ce}_{0.5} \mathrm{Yb}_{0.5} \mathrm{Fe}_{3.25} \mathrm{Co}_{0.75} \mathrm{Sb}_{12}$ pellets were collected using a Bruker D8 Advance Powder X-ray diffractometer, operating with Ge monochromated $\mathrm{Cu} \mathrm{K} \alpha 1$ radiation $(\lambda=1.54046 \AA)$ and fitted with a LynxEye detector. Data were collected over the angular range $5 \leq$ $2 \Theta /{ }^{\circ} \leq 90$ for 6 hours.

\section{Figure of merit ZT}

Seebeck coefficient $(\mathrm{S})$, electrical $(\sigma)$ and thermal $(\kappa)$ conductivities determine the figure of merit ZT $(\mathrm{ZT}=\mathrm{S} 2 \sigma \mathrm{T} / \kappa)$ of a material. A Linseis LSR-3 instrument was employed to measure simultaneously Seebeck coefficient and electrical conductivity over the temperature range $25 \leq \mathrm{T} /{ }^{\circ} \mathrm{C} \leq 550$ with a partial pressure of Helium. A Netzsch LFA 447 NanoFlash instrument was employed to determine the thermal conductivity of the samples over a temperature range of $25 \leq$ $\mathrm{T} /{ }^{\circ} \mathrm{C} \leq 300$ in $25^{\circ} \mathrm{C}$ steps. Higher Temperature measurements $(300 \leq$ $\left.\mathrm{T} /{ }^{\circ} \mathrm{C} \leq 550\right)$ in $50{ }^{\circ} \mathrm{C}$ steps were performed using an Anter Flashline 3000 instrument.

Page 3 of 7

\section{Measured thermoelectric properties of the skutterudite pellet}

X-ray diffraction based on Bragg's Law which explains that the cleavage faces of crystals appear to reflect X-ray beams at certain angles of incidence. It was used here to verify the crystal structure of the fabricated skutterudite thermoelectric materials. X-ray diffraction patterns of the two synthesized materials after consolidation by hotpressing present the basic reflections corresponding to the skutterudite structure and can be indexed in the cubic $\operatorname{Im} \overline{3}$ crystallographic space group. See Figure 4.

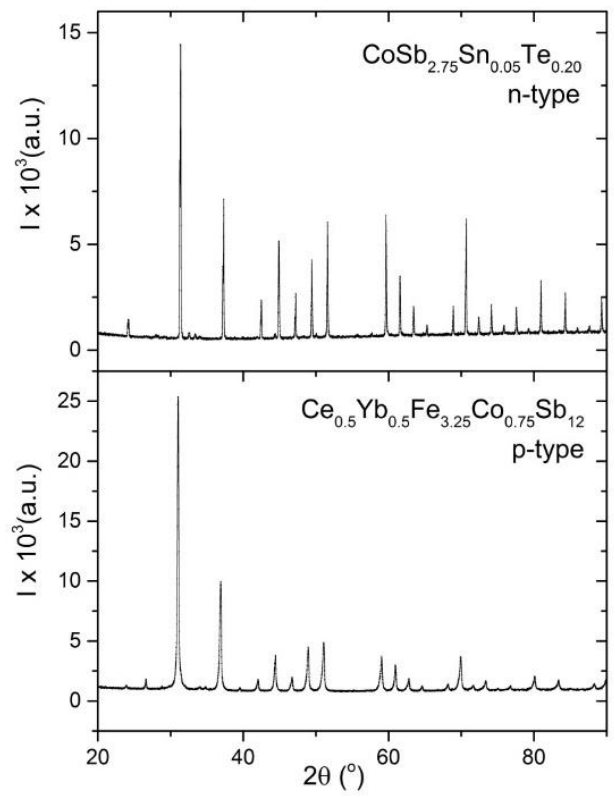

Figure 4. X-ray diffraction pattern of the skutterudite samples after consolidation by hot pressing.

The figure of merit ZT of the $\mathrm{n}$ and $\mathrm{p}$ thermoelectric materials versus the applied temperature is illustrated in Figure 5. It can be observed that the synthesized materials are appropriate for mid - high temperature range application, with maximum ZT of 1.13 at $405{ }^{\circ} \mathrm{C}$ for the $\mathrm{n}$-type material and 0.93 at $550{ }^{\circ} \mathrm{C}$ for the p-type material.

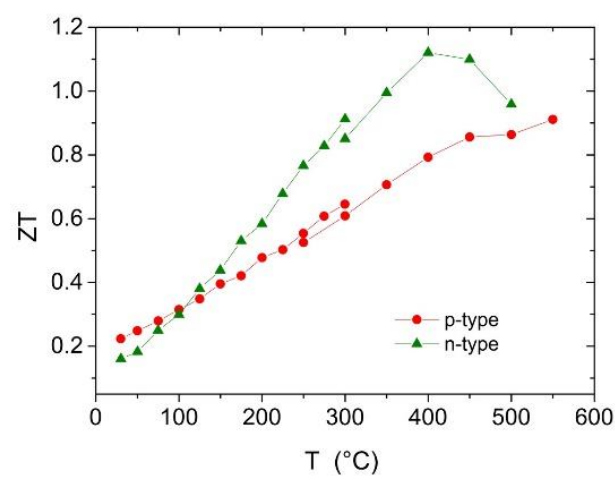

Figure 5. Temperature dependence of the figure of merit, ZT, of the skutterudites 


\section{Fabrication of Skutterudite Thermoelectric Module}

The characterized pellets manufactured in Reading University then was fabricated into TEM in Cardiff University in UK.

\section{Module Fabrication}

Modules consisting of 9 couples have been fabricated from $13 \mathrm{~mm}$ diameter skutterudite pellets which have the chemical composition $\mathrm{Co}_{1} \mathrm{Sb}_{2.75} \mathrm{Sn}_{0.05} \mathrm{Te}_{0.20}$ (n-type) and $\mathrm{Ce}_{0.5} \mathrm{Yb}_{0.5} \mathrm{Fe}_{3.25} \mathrm{Co}_{0.75} \mathrm{Sb}_{12}$, (p-type). The pellets are ground and polished to an equal height of approximately $1.5 \mathrm{~mm}$ and a metal diffusion barrier is then applied to the top and bottom surfaces via electroplating. This consists of a palladium layer followed by a nickel layer, with the total thickness of the layer verified to be approximately $10 \mu \mathrm{m}$ using a microscope. The pellets are then cut into legs using an Accutom-100 automated diamond cutting wheel, with each leg having the approximate dimensions $1.7 \mathrm{~mm}$ x $1.7 \mathrm{~mm} \times 1.5 \mathrm{~mm}$.

Two alumina ceramic plates ( $16 \mathrm{~mm} \times 13 \mathrm{~mm} \times 0.63 \mathrm{~mm})$ with bonded $2 \mathrm{~mm} \times 5 \mathrm{~mm} \times 0.35 \mathrm{~mm}$ copper strips (purchased from European Thermodynamics Ltd) are used to provide the thermal and electrical contacts. Junctions between the copper strips and the thermoelements are formed using the high temperature solder $\mathrm{Pb}_{93.5}$ $\mathrm{Sn}_{5} \mathrm{Ag}_{1.5}$. The joining materials have a melting point of around $296^{\circ} \mathrm{C}$, which restricts the hot-side temperature and is in reality a low temperature joining material for skutterudites, but is useful for basic characterization of the materials in a module formation. Lead based solders wet readily to the surface of the barrier layer and to the electrode and so a lower contact resistance is guaranteed. Also, the lower soldering temperatures makes inter-diffusion into the bulk less likely. All the components (i.e., thermoelements, alumina plates, and solder pieces) are sandwiched using a homemade assembly holder made from stainless steel. A good bond is achieved with the flux paste spread between both the thermoelement/solder and solder/Cu strip interfaces. The assembled module in the holder is then inserted into a quartz tube furnace (Carbolite furnaces) and isolated in an inert argon atmosphere. The holder is held in place until a temperature of $350^{\circ} \mathrm{C}$ is reached to melt the soldered joints. This ensured any difference between the temperature at the bond and at the thermocouple is mitigated. The holder is than removed and allowed to cool in an argon atmosphere. Finally, two contacting wires are soldered to the cold side of the module to provide the contacts. Figure 6 is the picture of this skutterudite TEM.

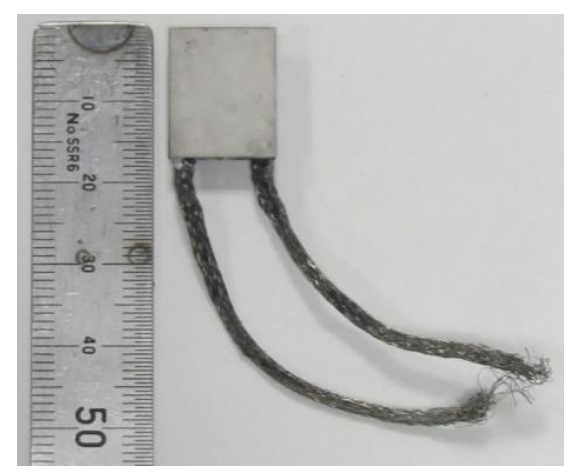

Figure 6. A picture of the skutterudite thermoelectric module.

\section{Module Testing}

Measurements are undertaken in a custom-made module testing vacuum system which is typically evacuated to approximately $5 \times 10-6$ mbar at the top of the turbo-pump. A copper block with inserts for electrical cartridge heaters is attached to the hot-side of the module while the cold-side is attached to a water cooled aluminum block which forms part of the vacuum chamber. The hot side heater block is approximately the same size as the module to minimize any thermal losses. A clamping plate is used to hold the heater against the module with $20 \mathrm{~mm}$ of thermal insulation to minimize thermal losses between the heater block and the plate. The plate is held in position by two screws embedded in the cold side and clamped in place using nuts and spring loaded washers to apply pressure. Both the module output power and heater input power measurements are taken using separate voltage and current leads to negate any resistances other than the module and its leads. Once the system has been evacuated, a B\&K Precision 9183 power supply is used to ramp the heater to a set temperature and to maintain a constant heat flow through the module. When the temperature is reached and has been stabilised, fast I-V curves are taken from open circuit to short circuit conditions using an Autolab potentiastat PGSTAT302N. The curve is taken with a scan rate of $5 \mathrm{~V} / \mathrm{s}$, to ensure that a constant temperature is maintained throughout the measurement. In all, five modules were fabricated and tested in this way. A module with characteristics similar to the average is then measured again to calculate the efficiency of the module. This measurement takes more time to complete, as it requires the module to be in thermal equilibrium at each point of the I-V curve. When current is allowed to flow through the module, additional heat is moved from the hot side to the cold side due to the Peltier effect (equation 6). Since the amount of heat supplied to the module is fixed, the result is a drop in the $\Delta \mathrm{T}$ across the module which is dependent on the amount of current flowing within the module.

\section{Results about the Module}

Figure 7 shows the nodule output power for the five modules with the coldside kept around $25^{\circ} \mathrm{C}$. The hotside was kept below the melting point of the solder as much as possible. Three of the five modules have a power output above the $1 \mathrm{~W}$ mark at $\Delta \mathrm{T}=300 \mathrm{~K}$ and this represents a power density of $0.48 \mathrm{Wcm}^{2}$. This high power density is possibly due to the leg length being quite short, which increases the power output at the expense of efficiency. This is reflected in the lower efficiency measured for module 4 . The efficiency of module 4 can be found in Figure 8. This module was selected from the five produced since its performance properties approximately match the average of the five. An efficiency value of around $3 \%$ at $\Delta \mathrm{T}=273 \mathrm{~K}$ was obtained, which is much lower than the value of $7 \%$ obtained by Salvador et al [24]. Their higher efficiency value is due to the much larger temperature difference across the module $(\Delta \mathrm{T}=460 \mathrm{~K})$. With a high temperature braze which can maintain a $500^{\circ} \mathrm{C}$ hotside temperature, it is expected that the skutterudite materials used in module 4 will achieve similar or better efficiency values at larger temperature difference.

The device used for measuring I-V curve has $\pm 0.2 \%$ uncertainty for both voltage and current measurement. Then according to the uncertainty analysis theory, the uncertainty of the computed electrical power shown in Figure 7 is about $\pm 0.283 \%$. 


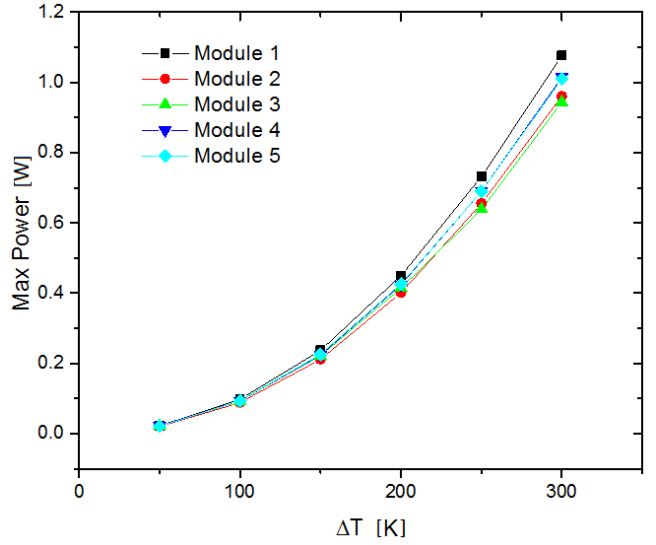

Figure 7. Electrical power output plotted against temperature difference across the module.

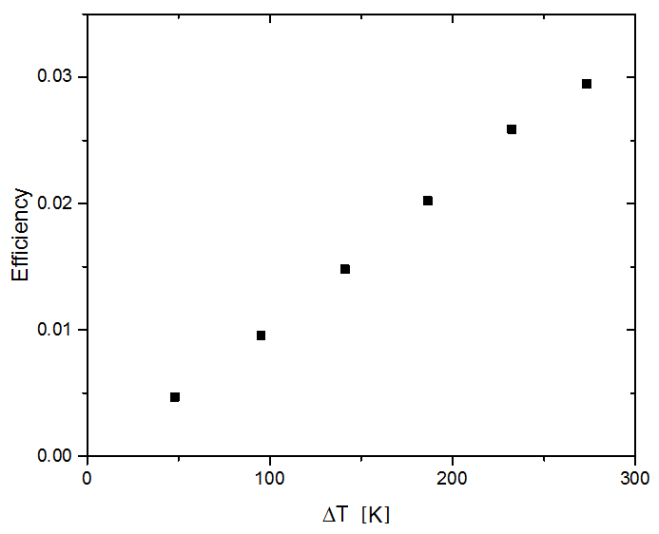

Figure 8. Module efficiency plotted as a function of temperature difference across the module. One module was chosen test the efficiency and module number 4 was selected since it represented the average performance properties of the five modules.

\section{Property Loss via Module Fabrication}

Three module thermoelectric properties which are module seebeck coefficient, module internal electric resistance and module thermal resistance can be estimated from measured pellet thermoelectric properties. These values were plotted together with the corresponding measured properties. They are shown in Figure 9 to Figure 11. Figure 9 shows that the seebeck coefficient was reduced from the module fabrication process. The reduction percentage increases with the delta temperature. The higher delta temperature, the higher loss of seebeck effect. Maximum percentage is about 23\%. Figure 10 shows that the module fabrication process increased the module internal electric resistance. The higher delta temperature, the higher percentage of the increased internal resistance. The maximum percentage of increased internal resistance is around $11 \%$. The module fabrication process has big impact on the module thermal resistance, See Figure 11. The reduction percentage is nearly constant at $44 \%$ for all delta temperature conditions.
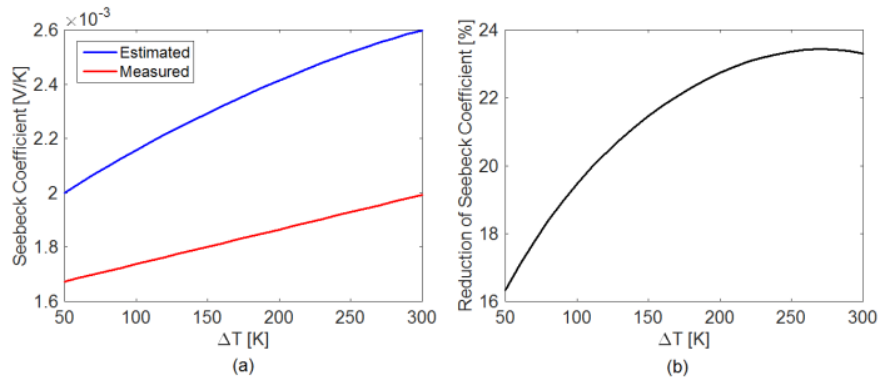

Figure 9. (a) Estimated and measured module seebeck coefficient; (b) Percentage of reduced seebeck coefficient.

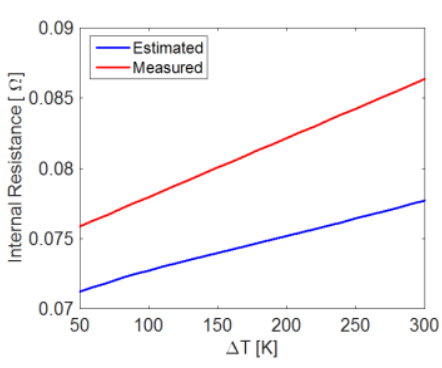

(a)

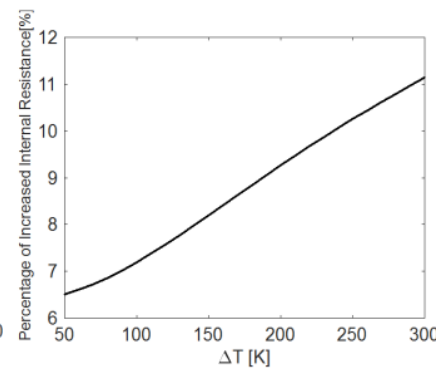

Figure 10. (a) Estimated and measured module internal electric resistance; (b) Percentage of increased electric resistance.

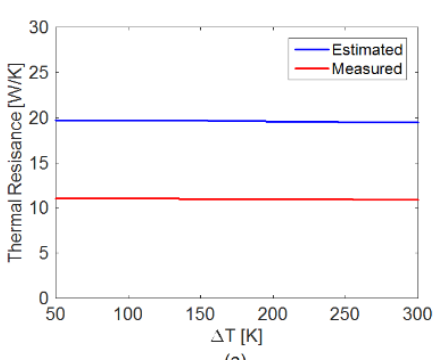

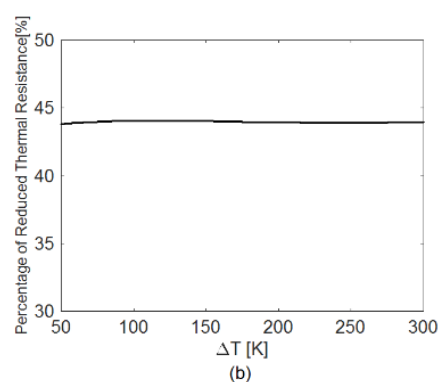

(b)
Figure 11. (a) Estimated and measured module thermal resistance; (b) Percentage of reduced thermal resistance.

The change of three thermoelectric properties caused by the module fabrication process all end up at property loss. The biggest property loss is thermal resistance. The second is the seebeck coefficient. Further investigation needs to be carried out to reduce the property loss of these three thermoelectric properties during the module fabrication process.

\section{Improved Thermoelectric Generator Performance Using Skutterudite Modules}

A MATLAB function based TEG dynamic model has been validated using engine test data. The model structure and validation results were discussed in another paper [25]. Use this model to estimate the maximum power output at steady state condition for a TEG device. Two TEG devices were assembled using two types of commercial modules which are ETL-TEM and HZ-TEM respectively. ETL-TEM and HZ-TEM are both made from Bismuth Telluride thermoelectric materials. But they have different leg length, module fill ratio and number of couples. The corresponding TEG devices were named as ETL-TEG and HZ-TEG in this paper. The third TEG device was 
using the skutterudite modules discussed in above then was named as sku-TEG. Three heat exchangers defined by different fin thermal resistances which are $0.05,0.15$ and 0.5 were used in the device level performance analysis for each TEG. For simplicity, the inlet gas flow rate was assumed at constant $60 \mathrm{~g} / \mathrm{s}$. By adjusting the gas in temperature, the hot side temperature of the first pair TEMs along the gas flow direction were kept around the module limit. The prediction results of three TEG devices above were compared in Figure 12 and Figure 13.

Figure 12 shows the selected gas in temperature and the results hot side temperature of the first pair TEMs for three TEG devices under three different hot side heat exchanger configurations. It can be seen from Figure 12 that the hot side temperature of the first pair TEMs for ETL-TEG and HZ-TEG are around $250^{\circ} \mathrm{C}$; the hot side temperature of the first pair TEGs for sku-TEG are around $300^{\circ} \mathrm{C}$. They are not precisely at $250^{\circ} \mathrm{C}$ and $300^{\circ} \mathrm{C}$, as the inlet gas in temperature was manually adjusted using the Simulink TEG model. However, this discrepancy does not affect the conclusions that are to be drawn by using this simulation results. It also can be seen from Figure 12 that the bigger the heat exchanger thermal resistance, the higher the allowable gas in temperature. This phenomena implies that hot side heat exchanger thermal resistance is not the smaller the better even without considering the influence of pressure drop. The design of the hot side heat exchanger should be optimized based on both pressure drop and exhaust gas conditions. Even though, ETLTEM and HZ-TEM has the same hot side temperature limit, ETLTEG allows higher gas in temperature than that of HZ-TEG. This gives the evidence that the module level design has big impact on device level performance.

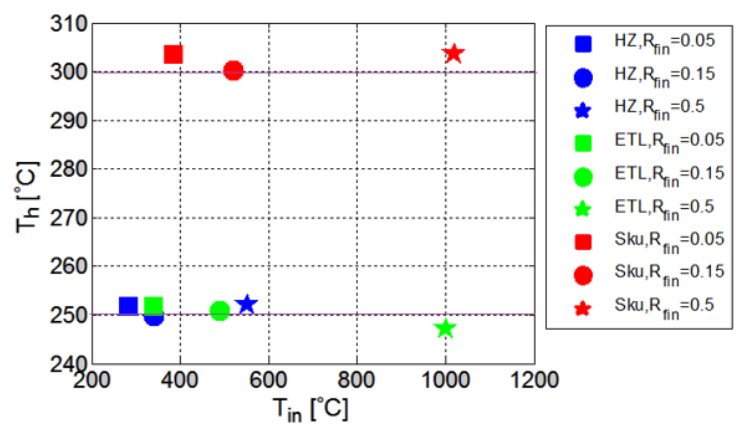

Figure 12. The maximum gas in temperature and hot side temperature of the first TEM

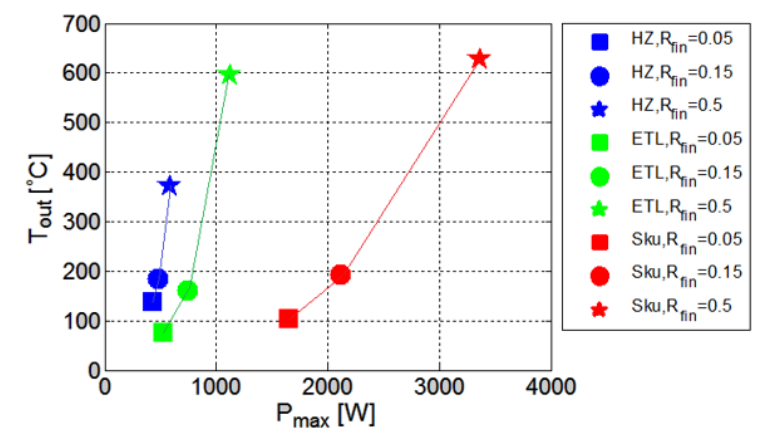

Figure 13. The maximum output of TEG and the gas out temperature of the TEG.

Page 6 of 7
The maximum gas in temperature for sku-TEG is slightly higher that that of sku-TEG under same fin thermal resistance conditions. See Figure 12. Figure 13 shows that sku-TEG has much higher output performance over that of ETL-TEG. According to authors' analysis, it is mainly due to the higher module thermal resistance, smaller module internal electrical resistance and better performance at high delta temperature of sku-TEM.

\section{Summary/Conclusions}

High temperature skutterudite thermoelectric materials enables higher temperature gas flow into the TEG. The skutterudite material was manufactured using ball milling method in University of Reading. The maximum ZT of this material is 1.13 at $405{ }^{\circ} \mathrm{C}$ for the n-type material and 0.93 at $550{ }^{\circ} \mathrm{C}$ for the p-type material. The thermoelectric modules using this skutterudite materials were fabricated in Cardiff University. These modules can work up to $400^{\circ} \mathrm{C}$ hot side temperature. The module testing results show there is property loss for all three thermoelectric properties during the module fabrication process. The biggest loss percentage is module thermal resistance which is around $44 \%$ for all delta temperature range. The second big property loss is seebeck coefficient, which is increases with the delta temperature. the maximum loss percentage is around $23 \%$ at about $300 \mathrm{~K}$ delta temperature. The internal electrical resistance was increased about $11 \%$ at $300 \mathrm{~K}$ delta temperature. Further investigation needs to be carried out to investigate the cause of the property loss and to reduce this loss. By using a validated TEG model, it was estimated that a TEG device that consists of skutterudite modules which can work at higher hot side temperature has much higher electrical power output. Further work needs to be done for experimental engine testing of a TEG device using these skutterudite modules.

\section{References}

1. Weerasinghea, W.M.S.R., Stobart, R.K., Hounshama, S.M., "Thermal Efficiency Improvement in Hight Output Diesel Engines A Comparison of a Rankine Cycle with Turbocompunding," Applied Thermal Engineering, Volumen 30, Issues 14-15, October 2010, Pages 2253-2256, doi:10.1016/j.applthermaleng.2010.04.028.

2. Hopmann, U., Algrain, M. C., "Diesel Engine Electric Turbo Compound Technology," SAE Technical Paper 2003-01-2294, 2003, doi: 10.4271/2003-01-2294.

3. Noor, A. M., Putch, R.C., Rajoo, S., "Waste Heat Recovery Technologies in Turbocharged Automotive Engine-A Review," Journal of Modern Science and Technology, Vol. 2 No. 1 March 2014, pp. 108-119.

4. Dolz, V., Novella, R., Garcia, A., Sanchez, J., "HD Diesel Engine Equipped with a Bottoming Rankine Cycle as a Waste Heat Recovery System. Part 1: Study and Analysis of the Waste Heat Energy," Applied Thermal Engineering, Volume 36, April 2012, Page 269-278.

5. Feru, E., "Auto-Calibration for Efficient Diesel Engines with a waste Heat Recovery System," 2015, CPI, Zutphen, The Netherlands.

6. J. Bass, N. Elsner and F. Leavitt, "Performance of the $1 \mathrm{~kW}$ Thermoelectric Generator for Diesel Engines", The International Conference on Thermoelectrics, 1994, Kansas City, Kansas, USA

7. Lan, S., Yang, Z., Stobart, R. and Winward, E., "The Influence of Thermoelectric Materials and Operation Conditions on the 
Performance of Thermoelectric Gnateaters for Automotive," SAE Technical Paper 2016-01-0219, doi: 10427/2016-01-0219.

8. M. Rosenberger, M. Deliner, M. Kluge, K. R. Tarantlk, "Vehicle Integration of a Thermoelectric Generator", Development Thermal Management, MTZ worldwide, April 2016, Volume 77, Issue 4,, pp 36-43

9. J. Bass, D. Krommenhoek, A. Kushch, S. Ghamaty, "Thermoelctric Generator Waste Heat Recovery System for Military Vehicles", 2004 DEER Conference, August 30, 2004

10. T. Kajikawa, " Overview of Progress in R\&D for Thermoelectric Power Generation Technologies in Japan”, 2012

11. A. Eder and M. Linde, "Efficient and Dynamic-The BMW Group Roadmap for the Application of Thermoelectric Generators", Conference, San Diego, January 3rd, 2011

12. Caillat, T., Fleurial, J. P., Snyder, G. J., et al., "A New High Efficiency Segmented Thermodelectric Unicouple", SAE Technical Paper 1999-01-2567, doi: 10427/1999-01-2567.

13. Bell, L. E., Science, 321 (2008) 1457-1461

14. Goldsmid, H. J. Introduction to thermoelectricity, 121 (2009). Springer

15. Ctirad, U. Skutterudite-Based Thermoelectrics. In Thermoelectrics Handbook: Macro to Nano. Rowe, D. M., Taylor \& Francis: (2005) 34-1 - 34-16

16. Uher, C. Semiconductors and semimetals, 69 (2001) 139-253

17. B. Orr, A. Akbarzadeh, M. Mochizuki, R. Singh A review of car waste heat recovery systems utilising thermoelectric generators and heat pipes Appl Therm Eng (2015) Volume 101, 25 May 2016, Pages 490-495.

18. M. Gao, D.M. Rowe, Ring-structured thermoelectric module, Semicond Sci Technol, 22 (2007), pp. 880-883.

19. Miner, A. 2014, "Industrialization of Thermoelectrics: Materials, Modules and Manufacturing Scalability", 4th Thermoelectrics IAV Conference presentation, viewed 10 October 2016, http://romny-scientific.com/technology/

20. Oftedal, I. Norsk, Geol. Tidssk 8 (1928): 250-257

21. Sales, B. C., Mandrus, D., \& Williams, R. K., Science, 272 (1996) 1325-1328

22. Rull-Bravo, M.; Moure, A; Fernández, J.F.; Martín-González, M., RSC Advances, 5 (2015) 41653-41667

23. Gao Min, "Thermoelectric Energy Harvesting”, Chapter 5, in "Energy harvesting for autonomous systems", Ed. Beeby and White, Artech House Inc., pp135-157, 2010.

24. Salvador, J.R., Cho, J.Y., Ye, Z. et al. Journal of Elec Materi (2013) 42: 1389. doi:10.1007/s11664-012-2261-9

25. Yang, Z., Song, L., Stobart, R., "A Comparison of Four Modelling Techniques for Thermoelectric Generator," submitted to SAE Congress 2017.

26. Chen, S., Lukas, K., Liu, W., Opeil, C., Chen, G., Ren, Z., "Effect of Hf Concentration on Thermoelectric Properties of Nanostructured N-Type Half-Heusler Materials HfxZr1xNiSn0.99Sb0.01", Advanced Energy Materials, May, 2013, DOI: 10.1002/aenm.201300336

\section{Contact Information}

Zhijia Yang, Aeronautical \& Automotive Engineering Department, Loughborough University, z.yang2@lboro.ac.uk
Jesús Prado-Gonjal, Chemistry Department, University of Reading, j.pradogonjal@reading.ac.uk

Matthew Phillips, Cardiff University, PhillipsM19@cardiff.ac.uk

Song Lan, Aeronautical \& Automotive Engineering Department, Loughborough University, s.lan@lboro.ac.uk

Anthony Powell, Chemistry Department, University of Reading, a.v.powell@reading.ac.uk

Paz Vaqueiro, Chemistry Department, University of Reading, p.vaqueiro@reading.ac.uk

Min Gao, School of Engineering, Cardiff University, $\underline{\text { min@ cardiff.ac.uk }}$

Richard Stobart, Aeronautical \& Automotive Engineering Department, Loughborough University, r.k.stobart@lboro.ac.uk

Rui Chen, Aeronautical \& Automotive Engineering Department, Loughborough University, r.chen@lboro.ac.uk

\section{Acknowledgments}

The authors would like to gratefully acknowledge the UK Engineering and Physical Sciences Research Council (EPSRC) for funding this research work under Grant Number: EP/K026658/1.

\section{Definitions/Abbreviations}

ETC

Electrical-Turbo-

TC Compounding

TEG

TEM Turbo-Compounding

Thermoelectric Generator

Thermoelectric Module

ORC

Page 7 of 7

$10 / 19 / 2016$ 